\title{
Severe eye damage in infants with microcephaly is presumed to be due to Zika virus
}

\author{
Michael McCarthy
}

Seattle

A Brazilian study of 29 infants with microcephaly and a presumed diagnosis of congenital Zika virus infection has found that a third had vision threatening eye damage.

In the study Rubens Belfort, of the Federal University of São Paulo, and colleagues examined infants born with microcephaly after a severe outbreak of Zika virus infections in Salvador, in the northeastern state of Bahia.

All of the infants had a head circumference of $32 \mathrm{~cm}$ or less at birth. Of their mothers, 23 (79.9\%) had signs and symptoms consistent with Zika virus infection during pregnancy, including rash, fever, arthralgia, and headache, but none reported signs or symptoms of conjunctivitis. Zika virus infections are often asymptomatic, as only about one in five of those infected develops symptoms.

Infants were excluded from the study if they had a familial history of microcephaly or if the mother had a history of alcohol or illicit drug use during pregnancy. Other congenital infections such as toxoplasmosis, rubella, cytomegalovirus, herpes simplex virus, or HIV were ruled out.

Ocular abnormalities were reported in 10 of the 29 infants (34.5\%). The most common lesions were focal pigment mottling and chorioretinal atrophy that tended to be located in the posterior pole of the eye, especially the macular area. Other lesions included optic nerve involvement, including optic nerve hypoplasia and severe optic disc cupping, lens subluxation, and one case of bilateral iris coloboma. No infants had vasculitis or active uveitis.

The findings showed that pregnant women with few or no symptoms of Zika virus infection may give birth to microcephalic newborns with ophthalmoscopic lesions, the researchers wrote, adding, "An important question is whether patients without microcephaly will need to be screened to identify these ocular lesions."

They concluded, "Infants with microcephaly should undergo routine ophthalmologic evaluations to identify such lesions. In high transmission settings, such as South America, Central America, and the Caribbean, ophthalmologists should be aware of the risk of congenital ZIKV [Zika virus]-associated ophthalmologic sequelae."

In an invited commentary ${ }^{2}$ Lee M Jampol and Debra A Goldstein, of Northwestern University Feinberg School of Medicine in Chicago, USA, noted that, although Brazil has seen a 20-fold increase in reported cases of microcephaly since the Zika virus outbreak began, the association remains presumptive because definitive serologic testing for the virus was not available at the time of the outbreak.

However, Jampol and Goldstein concurred with Belfort and colleagues that, in areas where Zika virus is present, clinicians should perform ophthalmologic exams on all microcephalic babies. They wrote, "Because it is still unclear whether the eye lesions occur in the absence of microcephaly, it is premature to suggest ophthalmic screening of all babies born in epidemic areas."

1 De Paula Freitas B, de Oliveira Dias JR, Prazeres J, et al. Ocular findings in infants with microcephaly associated with presumed Zika virus congenital infection in Salvador, Brazil. JAMA Ophthalmol 2016; published online 9 Feb, doi:10.1001/jamaophthalmol.2016.0267.

2 Jampol LM, Goldstein DA. Zika virus infection and the eye. JAMA Ophthalmol 2016; published online 9 Feb, doi:10.1001/jamaophthalmol.2016.0284. 